\title{
The Rare Case of Moyamoya Disease Initially Suspected with Transcranial Doppler Real-Time Color Flow Imaging Study
}

\author{
Alexander Sosnovsky ${ }^{1,4}$, Eric Hirsch ${ }^{1,2}$, Erica Glants ${ }^{1}$, Tahir Sheikh ${ }^{1}$, Daryl Duran ${ }^{1}$, Alena Mayorchyk ${ }^{1}$, Alexander \\ Sosnovskiy ${ }^{1}$ and Ranga Krishna ${ }^{1,3}$
}

${ }^{1}$ Total Neuro Care 1513 Voorhies Avenue, USA

${ }^{2}$ SUNY Downstate College of Medicine 395 Lenox Road Brooklyn, USA

${ }^{3}$ New York Community Hospital 2525 Kings Highway, USA

${ }^{4}$ NYU College of Arts \& Sciences, 100 Washington Square East, USA

Submission: June 18, 2018; Published: August 14, 2018

*Corresponding author: Eric Hirsch, Total Neuro Care 1513 Voorhies Avenue, Brooklyn NY, East 10th Street, Brooklyn N.Y. 11230,

Tel: 516-668-2310 1075; Email: eric.hirsch@downstate.edu

Abstract

A 35-year-old Hispanic female presented to the neurology clinic with a four-year history of severe, aura-like headaches beginning after a motor vehicle accident. She was previously diagnosed with a primary migraine disorder and prescribed verapamil but failed to improve. On history-taking, the patient described a distant episode consistent with TIA, and transcranial Doppler was performed to screen for underlying vascular pathology. Ultrasonography revealed flow changes in the circle of Willis suspicious for moyama disease. The diagnosis was subsequently confirmed on MRA.

This case illustrates the complex clinical picture of moyamoya disease and the utility of Transcranial Doppler Real-time color flow imaging in diagnosis. Diagnosis was challenging due to the unusual circumstances surrounding onset, the non-specific presentation, and the fact that the patient did not fit the "classic" East-Asian demographic. Careful history-taking was required to elicit evidence of vascular pathology. Although MRA is widely considered "first line" for diagnosis of moyamoya disease, Doppler offers an affordable, accessible, and informative modality for initial assessment of patients with a suspicion for moyamoya disease. It is important for clinicians to be aware of this modality as a screening tool for moyamoya.

Keywords: Moyamoya; Ultrasound; Hispanic female; Doppler; Neurology; Clinic; Headache; Disease; Ultrasound; Bilateral stenosis; Proximal branches; Brain; Japanese; Investigators; Chronic ischemia; Numerous fragile; Smoke; Ganglia; Thalamus; Velocity; Ambiguous evidence; Bidirectional

\section{Introduction}

Moyamoya disease is a rare occlusive vasculopathy of the circle of Willis. The disease is characterized by progressive, bilateral stenosis of the intracranial ICA and its proximal branches. In the setting of chronic ischemia, numerous fragile collateral vessels develop around the base of the brain. Early Japanese investigators likened the hazy appearance of this collateral vasculature on angiography to a "moyamoya" (puff of smoke), giving the disease its name. When a moyamoya-like pattern of obstruction and collateral-formation develops in the setting of another condition (e.g. atherosclerosis), it is called moyamoya syndrome. The term moyamoya disease is reserved for cases in which stenosis occurs bilaterally in the absence of an inciting condition [1].
The causes of moyamoya disease are still being investigated. While it is known that vessel occlusion results from a combination of hyperplasia of smooth-muscle cells and luminal thrombosis, this process may have multiple pathophysiological antecedents $[1,2]$. Genetic factors appear to play a role in some patients; the proportion of patients who have affected first-degree relatives is reported to be $10 \%$ in Japan, while one U.S. study found a rate of 6\% [2] Several susceptibility loci are known (e.g. 3p, 6p, $17 q)$ and a recent study has identified RNF213 as the first gene associated with moyamoya [2].

The clinical picture of moyamoya disease is complex. Patients can present with a broad spectrum of neurological impairments arising from global cerebral hypoperfusion, focal infarcts, and 


\section{Open Access Journal of Neurology \& Neurosurgery}

the hemorrhage of fragile collaterals. The disease has two peak incidence periods in the first and fifth decades of life, with key differences in presentation between pediatric and adult patients [1]. Pediatric moyamoya disease has almost exclusively an ischemic picture, with complications ranging from acute episodes of stroke and TIA to the progressive development of headaches, seizures, focal deficits, and developmental delay. While adult patients also present with ischemic symptoms, up to $40 \%$ have superimposed intracranial hemorrhage [1,3]. Bleeding typically occurs in the frontal horn of the lateral ventricle, basal ganglia, and thalamus, leading to severe headache, vomiting, and focal neurological deficits. Because it is a rare cause of complicated headache syndrome with a variable symptomatology, moyamoya can masquerade as migraine.

\section{Case Report}

A 35-year-old Hispanic female presented to the neurology clinic with a four-year history of migraine-like headaches. She said that she had suffered migraines for a period in her 20s, but that they gradually stopped without clinical intervention. Four years ago, she was in a car accident, sustaining multiple disc herniations with severe radiculopathy. She subsequently developed chronic pain refractory to physical therapy and symptoms of PTSD. Immediately after the accident, her migraines returned in a more severe and persistent form. She described the headaches as a pan-cranial "pounding" with "a sense of pressure," accompanied by photophobia and an aura of increasing leftsided visual loss. They occurred almost daily, lasting several hours in the morning and improving in the afternoon, without complete resolution. She was prescribed verapamil for migraine prophylaxis but experienced no improvement. There was brief relief with fioricet and heavy use of recreational cannabis. She said that the headaches were becoming progressively more severe, particularly in the last 7 months, and substantially impacting her quality of life. In addition to headaches, the patient reported persistent dizziness, loss of balance, and disorganized thought (for which she had been prescribed Risperdal $2 \mathrm{mg}$ ). She also described a particularly severe episode of lightheadedness and dizziness that occurred three months prior to her accident. Her description of the episode was possibly consistent with TIA; however, she did not seek medical attention at the time. She denied any other significant past medical history. There was no family history of headache disorder or cerebrovascular disease.

On neurological exam, the patient was distressed but otherwise attentive and cooperative. There was subjective dizziness with neck flexion and extension and unsustained nystagmus with positive hallpike maneuver. There were no other signs of neurological dysfunction. Although the patient's symptoms could have been consistent with a primary migraine disorder, their progressive course in the absence of any apparent predisposing factor prompted us to look for a secondary cause. The history of a TIA-like episode raised suspicion for a vascular abnormality. Real-time transcranial Doppler color flow imaging study was performed to assess for vascular pathology.

\section{Results}

The sonographic study revealed changes consistent with obstructive vasculopathy in the circle of Willis. There was low resistance flow bilaterally in the proximal middle cerebral arteries with significantly decreased velocity on the right side and bidirectional disorganized flow on the left side and very low resistance flow in the left distal middle cerebral artery (Figure 1 \& 2). Collateral flow with elevated velocity was detected in the right middle cerebral artery area. The right terminal internal carotid artery was not seen. The right anterior cerebral artery showed significant flow velocity reduction and bidirectional flow. On the left side, a tangled cluster of dilated blood vessels was seen on Color and PW Doppler at the terminal internal carotid artery/proximal middle cerebral artery level with disorganized blood flow, significantly elevated velocities, and bruit. Significantly increased velocity and bruit were seen in the left anterior cerebral artery. Mildly elevated velocities were noted in both vertebral arteries and the basilar artery. These findings were interpreted as ambiguous evidence. Very low velocities and resistance of flow in the right middle and anterior cerebral arteries, along with the presence of high velocity collateral flow and absence of flow detected in the terminal internal carotid artery, were suggestive of the right terminal internal carotid artery obliteration. Dilated tangled blood vessels at the left terminal internal carotid artery/proximal middle cerebral artery level with elevated velocities, bruit and bidirectional disorganized flow indicated high grade stenosis with collateral flow versus a small arteriovenous malformation. Extremely high velocity in the left anterior cerebral artery was suggestive of high-grade stenosis versus vasospasm. Mildly elevated velocities in the vertebral arteries and the basilar artery indicated possible compensatory flow to the brain through the basilar artery.

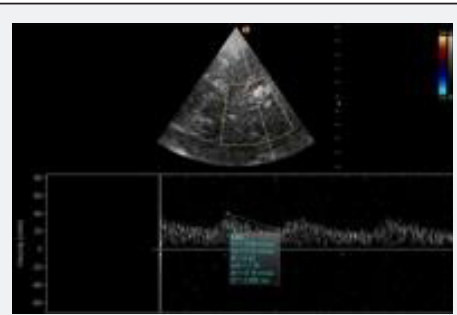

Figure 1: Low resistance flow in the right proximal middle cerebral artery with significantly decreased velocity.

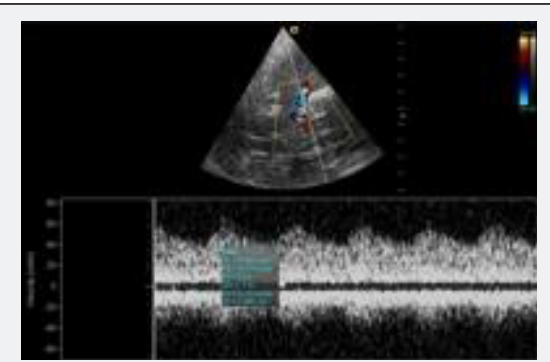

Figure 2: Low resistance bidirectional disorganized flow in the left proximal middle cerebral artery. Tangled cluster of dilated blood vessels visible at the left terminal internal carotid artery/ proximal middle cerebral artery level. 


\section{Open Access Journal of Neurology \& Neurosurgery}

These ultrasound findings were highly suggestive of moyamoya disease. The patient was referred to the emergency department for advanced imaging studies. MRA revealed bilateral tapering of the internal carotid artery with almost complete occlusion of the termini and collateral vessels at the base of the skull (Figure 3). MRI revealed numerous foci of abnormal white matter consistent with small ischemic lesions. These findings were consistent with a diagnosis of chronic ischemia due to moyamoya disease. Surgical revascularization was performed, after which the patient experienced nearly complete resolution of her headaches. She was placed on aspirin therapy and monitored for recurrence.

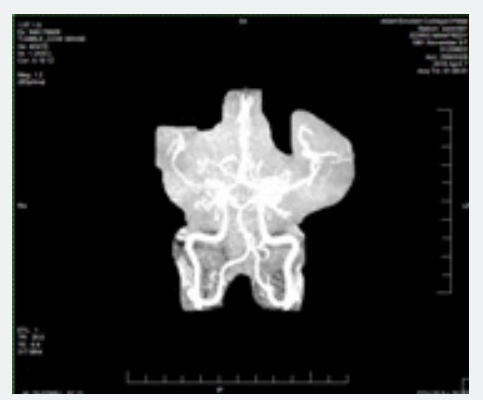

Figure 3: Coronal MRA showing stenosis of the terminal ICA with collateral circulation.

\section{Discussion}

This case illustrates the complex clinical picture of moyamoya disease and the utility of Transcranial Doppler Real-time color flow imaging in diagnosis. Our patient suffered from debilitating headaches for four years before moyamoya was first suspected on Doppler. Moyamoya has been reported as a rare cause of migraine with aura-like headaches [4]. In this case, diagnosis was undoubtedly complicated by the onset after a car accident in the setting of multiple traumatic sequelae. Extreme physiologic stress associated with the accident most likely worsened global hypoperfusion and precipitated the development of symptomatic ischemia. While MRA is widely considered the firstline diagnostic tool for moyamoya, the efficacy of ultrasound imaging has been well-attested [1,5,6]. Doppler offers several advantages over MRA as a first-line screening technique for moyamoya disease, including greater accessibility and lower cost. It can also have added prognostic value in quantifying the severity of blood flow changes. It is important for clinicians to be aware of this modality as a screening test for moyamoya as well as the characteristic findings associated with the disease.

In this case, diagnosis may have been further complicated by the patient's Hispanic ethnicity. Moyamoya is a rare disease classically associated with an East Asian demographic. However, the true demographic picture in the United States is highly varied. One study of 2280 admissions for moyamoya disorder found demographics of white (49\%), black (24\%), Hispanic (11\%), Asian $(11 \%)$, and other $(3.3 \%)$, suggesting that a moyamoya patient in the United States is just as likely to be Hispanic as she is to be Asian [3]. With the intermingling of the global gene pool, diseases that were previously defined by a single ethnic demographic are becoming increasingly prevalent in the general population. Moyamoya disease is an important example of this trend.

\section{Conclusion}

Moyamoya disease is a rare, but significant cause of complicated headache syndrome across multiple ethnic groups in the United States. Transcranial Doppler Real-time color flow imaging offers an affordable, accessible, and informative modality for initial assessment of this condition.

\section{References}

1. Fujimura M, Sonobe S, Nishijima Y, Niizuma K, Sakata H, et al. (2014) Genetics and biomarkers of moyamoya disease: significance of RNF213 as a susceptibility gene. J Stroke 16(2): 65-72.

2. Scott RM, Smith ER (2009) Moyamoya disease and moyamoya syndrome. N Engl J Med 360(12): 1226-1237.

3. Starke RM, Crowley RW, Maltenfort M, Jabbour PM, Gonzalez LF, et al. (2012) Moyamoya disorder in the United States. Neurosurgery 71(1): 93-99.

4. Park-Matsumoto YC, Tazawa T, Shimizu J (1999) Migraine with auralike headache associated with moyamoya disease. Acta Neurol Scand 100(2): 119-121.

5. Murata T (2014) Usefulness of the Time-Resolved Three-Dimensional MRA (4D-MRA) in Moyamoya Disease. European Congress of Radiology.

6. Takase K, Kashihara M, Hashimoto T (1997) Transcranial Doppler ultrasonography in patients with moyamoya disease. Clin Neurol Neurosurg 99: S101-S105.

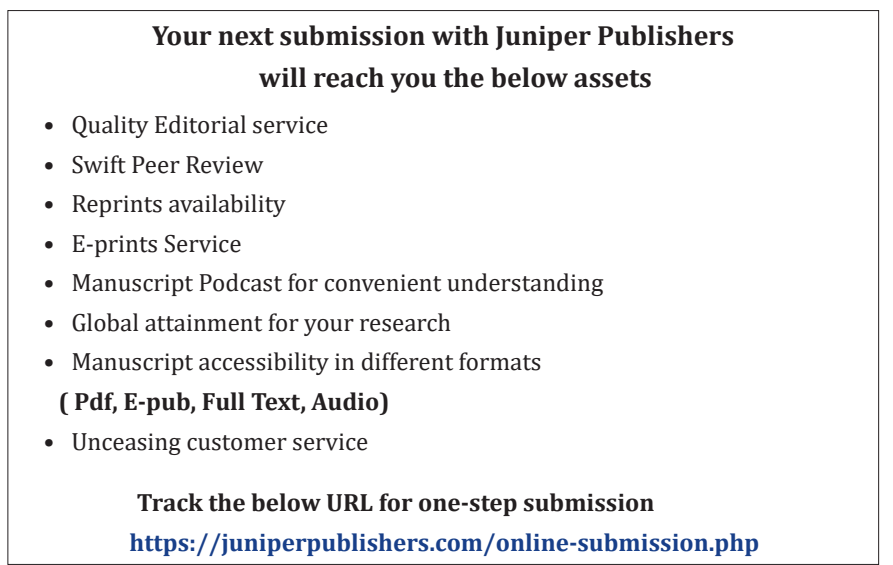

